\title{
Propósitos y Representaciones, revista de Psicología educativa, indizada en SciELO Perú
}

\author{
Propositos y Representaciones, Journal of Educational \\ Psychology now indexed in Peruvian Scientific Electronic \\ Library Online
}

Jorge Rodríguez-Sosa (D) Universidad San Ignacio de Loyola, Lima, Perú. ORCID: https://orcid.org/0000-0002-8440-4891

Joel Alhuay-Quispe ${ }^{\text {iD }}$ Universidad San Ignacio de Loyola, Lima,Perú. Universidad Nacional Mayor de San Marcos ${ }^{1}$, Lima, Perú. ORCID: http://orcid.org/0000-0002-1903-4687

Ronald M. Hernández* (iD Universidad San Ignacio de Loyola, Lima, Perú. Universidad de San Martín de Porres, Lima, Perú. ORCID: http://orcid.org/0000-0003-1263-2454

Josmel Pacheco-Mendoza (iD) Universidad San Ignacio de Loyola, Lima, Perú ORCID: http://orcid.org/0000-0002-2251-8092

*Correspondencia

Email: rhernandezv@usil.edu.pe

\section{Nota:}

${ }^{1} \mathrm{El}$ autor es miembro de los grupos de investigación "Tecnologías, Derechos y Libertades Informativas (TDLI)" y "Media Lab UNMSM" adscritos a Facultad de Letras y Ciencias Humanas UNMSM.

\section{Citar como:}

Rodríguez-Sosa, J., Alhuay-Quispe, J., Hernández, R.M., \& Pacheco-Mendoza, J. (2018). Propósitos y Representaciones, revista de Psicología educativa, indizada en SciELO Perú. Propósitos y Representaciones, 6(1), 09-19 doi: http://dx.doi.org/10.20511/pyr2018.v6n1.213

(C) Universidad San Ignacio de Loyola, Vicerrectorado de Investigación, 2018

(cc) BY-NC-ND Este artículo se distribuye bajo licencia CC BY-NC-ND 4.0 Internacional (http://creativecommons.org/licenses/by-nc-nd/4.0/). 
Palabras clave: Revistas científicas, Psicología de la educación, SciELO Perú, Impacto científico.

Publicado el volumen 6 - número 1, y luego de un quinquenio de existencia de la revista, a quienes somos miembros del equipo responsable de los procesos editoriales de Propósitos y Representaciones nos parece pertinente reflexionar sobre lo trabajado y logrado en estos años, principalmente en dos campos específicos: el proceso de indización de la revista y la visibilidad de sus publicaciones.

Desde la publicación del primer número en el 2013, Propósitos y Representaciones ha mantenido una periodicidad continua y una gestión editorial de calidad. Este proceso permitió alcanzar importantes calificaciones e indizaciones en plataformas como: DOAJ (Directory of Open Access Journals), un importante directorio global que proporciona acceso abierto a revistas en línea de alta calidad y revisadas por pares; DIALNET, un portal bibliográfico que prioriza la literatura científica hispana con énfasis en Ciencias Humanas, Sociales y Jurídicas; REDIB, un agregador de repositorios de revistas que incluye contenidos científicos y académicos en formato electrónico producidos en el ámbito iberoamericano; ERIC (Education Resources Information Center), una base de datos bibliográfica referente mundial de la investigación de habla inglesa sobre educación; ESCI (Emerging Sources Citation Index), una nueva colección de Web of Science que incluye revistas científicas en postulación a formar parte de alguna de las colecciones del Core de Clarivate Analytics; y SciELO Perú, una biblioteca virtual de revistas científicas peruanas que es parte de la red scielo.org. Adicionalmente, la revista ha sido integrada a otras 14 plataformas como agregadores de información, bases de datos bibliográficas y buscadores académicos, como Google Scholar y CrossRef.

En cuanto a visibilidad web y posicionamiento académico de una revista, la plataforma tecnológica que emplea y la calidad de sus contenidos son factores determinantes para alcanzar un desempeño diferenciador frente antes tantas revistas electrónicas existentes. Es ese sentido, es necesario 
que el equipo editorial de una revista tenga en cuenta que Google y Google Scholar se han convertido en la puerta de entrada para los usuarios que buscan información actualizada y académica (Alhuay-Quispe, QuispeRiveros, Bautista-Ynofuente \& Pacheco-Mendoza, 2017, p. 245).

A continuación, presentamos un panorama general sobre la visibilidad web e impacto científico que arroja Google Scholar sobre Propósitos y Representaciones. Los datos obtenidos con el software Publish or Perish (Harzing, 2007) sugieren que una "ventana de exposición" de tres años es necesaria para que los artículos empiecen a ser citados con cierta frecuencia (Tabla 1). Una vez que se supera los tres años, la cantidad de citaciones se incrementa exponencialmente, al margen de la cantidad de documentos o de números disponibles, la aparición en plataformas de clasificación de revistas o bases de datos bibliográficas (de temas especializados o de campos diversos), e incluso del idioma en que se publica una revista.

La Tabla 1 muestra que los artículos publicados el 2013, que son 18 documentos alcanzan 70 citas, mientras que 21 documentos publicados el 2017 solo 10 citas, en una situación opuesta entre los años de exposición del documento y su impacto, sin que el número de documentos juegue un rol de intervención; el mismo efecto de "ventana de exposición" ocurre en el ratio de conteo fraccionado de citas por autor (ci/au) y citas por artículo publicado (ci/doc).

\section{Tabla 1.}

Citas por años según Google Scholar

\begin{tabular}{llllll}
\hline Años & Doc & Citas & Autores & Ci/Au & Ci/Doc \\
\hline 2013 & 18 & 70 & 31 & 2.26 & 3.89 \\
2014 & 14 & 55 & 19 & 2.89 & 3.93 \\
2015 & 16 & 40 & 29 & 1.38 & 2.50 \\
2016 & 20 & 36 & 29 & 1.24 & 1.80 \\
2017 & 21 & 10 & 14 & 0.71 & 0.48 \\
\hline
\end{tabular}


El medio o plataforma en el que aparecen las publicaciones de Propósitos y Representaciones muestran una aproximación con el comportamiento del impacto científico. Para el caso de Dialnet, muestra que los artículos en español publicados obtienen un ratio de citación por documento (ci/doc) de 1,12; mientras que ERIC, que solo incluye artículos publicados en inglés, arroja un ratio de 0.21 (Tabla 2).

A partir de lo que se muestra en la Tabla 2, podríamos concluir que los principales lectores y autores citantes de la revista provienen de habla hispana o con interés en temas psicológicos y pedagógicos de la realidad latinoamericana. Por otro lado, resulta importante notar que los artículos visibles en Google Scholar a través del portal OJS obtienen considerable cantidad de citas, superados ligeramente por aquellos visibles a través de Dialnet, lo que indica que la inclusión de la revista en esta plataforma fue una ventaja para el posicionamiento e impacto científico alcanzado.

\section{Tabla 2.}

Citas por documentos según la fuente e idioma de los artículos

\begin{tabular}{llcccc}
\hline Fuente & Idioma & Años & Doc & Citas & Ci/Doc \\
\hline dialnet.unirioja.es & spa & $2013-2017$ & $76^{*}$ & 85 & 1.12 \\
revistas.usil.edu.pe & spa, eng & $2013-2017$ & 89 & 93 & 1.04 \\
search.proquest.com & spa, eng & $2013-2017$ & 89 & 21 & 0.24 \\
eric.ed.gov & eng & $2014-2017$ & $56^{* *}$ & 12 & 0.21 \\
\hline
\end{tabular}

*Excluye: Editorial, y Obituarios.

**Solo incluye: Artículos de investigación, y Artículos de revisión.

Dentro de los futuros retos de la gestión editorial de Propósitos y Representaciones se encuentra el de continuar con el proceso de indización en plataformas multidisciplinarias o en bases especializadas y de alto impacto, con el propósito de mejorar significativamente el posicionamiento de la revista y el impacto de sus publicaciones. Los esfuerzos siguen dirigiéndose hacia el logro de una visibilidad internacional, incentivando la generación y 
divulgación de estudios científicos en el campo de la psicología educativa (Hernández, 2018).

\section{Referencias}

Alhuay-Quispe, J., Quispe-Riveros, D., Bautista-Ynofuente, L., \& PachecoMendoza, J. (2017). Metadata Quality and Academic Visibility Associated with Document Type Coverage in Institutional Repositories of Peruvian Universities. Journal of Web Librarianship, 11(3-4), 241254. Doi: https://doi.org/10.1080/19322909.2017.1382427

Harzing, A.W. (2007). Publish or Perish. Recuperado de: http://www. harzing.com/pop.htm

Hernández, R. M. (2018,). Producción científica latinoamericana en la revista Anales de Pediatría. Anales de Pediatría. Doi: https://doi.org/10.1016/j. anpedi.2017.12.012 